\title{
BMJ Open Duration of untreated psychosis and pathways to care in Suriname: a qualitative study among patients, relatives and general practitioners
}

\author{
Atousa van Beek (D) ,1,2 Janine de Zeeuw, ${ }^{3}$ Menno de Leeuw, ${ }^{1,2}$ Mia Poplawska, ${ }^{1,2}$ \\ Lise Kerkvliet, ${ }^{1,2}$ Rudi Dwarkasing, ${ }^{4}$ Randhir Nanda, ${ }^{4}$ Wim Veling ${ }^{2}$
}

To cite: van Beek A, de Zeeuw J, de Leeuw M, et al. Duration of untreated psychosis and pathways to care in Suriname: a qualitative study among patients, relatives and general practitioners. BMJ Open 2022;12:e050731. doi:10.1136/ bmjopen-2021-050731

- Prepublication history and additional supplemental material for this paper are available online. To view these files, please visit the journal online (http://dx.doi.org/10.1136/ bmjopen-2021-050731)

Received 26 February 2021 Accepted 22 November 2021

Check for updates

(C) Author(s) (or their employer(s)) 2022. Re-use permitted under CC BY-NC. No commercial re-use. See rights and permissions. Published by BMJ.

${ }^{1}$ Faculty of Medical Sciences, University of Groningen, Groningen, The Netherlands ${ }^{2}$ Department of Psychiatry, University Medical Centre Groningen, Groningen, The Netherlands

${ }^{3}$ Department of Health Sciences, Global Health Unit, University Medical Centre Groningen, Groningen, The Netherlands ${ }^{4}$ Psychiatric Centre Suriname, Paramaribo, Suriname

Correspondence to Dr Janine de Zeeuw; j.de.zeeuw@umcg.nl

\section{ABSTRACT}

Purpose Psychosis is a severe mental health problem and is responsible for poor health outcomes, premature mortality and morbidity, especially in low- and middleincome countries. The duration of untreated psychosis (DUP), that is the time period between onset of symptoms until initiation of appropriate treatment by a healthcare professional, is one of the main determinants for successful treatment in western settings. This study aims to explore the factors related to the DUP among Surinamese patients using the perspectives from patients, their families and first-line healthcare professionals in Suriname.

Methods Semi-structured interviews were conducted with patients having a history of psychosis, family members and general practitioners between February 2019 and April 2019 in Suriname. Interviews were taperecorded and transcribed verbatim. Data were analysed using a thematic analysis for which an inductive and deductive approach was applied.

Results In total, 28 patients, 13 family members and 8 general practitioners were interviewed. Five patients were excluded from the study. A median DUP of 4 months was found (IQR 1-36). Identified themes related to DUP included presentation of symptoms and illness awareness, help-seeking behaviour and alternative medicine, social support and stigma, financial and practical factors. Conclusion Multiple factors were related to DUP, of which poor illness awareness, traditional medicine, stigma and social support were predominant. Poor illness awareness and use of alternative medicine were related to a longer DUP. Stigma was often an obstacle for patients and their families. Social and family support was important in helping patients to get medical help sooner. Other explored factors including financial and practical factors did not contribute to DUP.

\section{INTRODUCTION}

Globally, mental and addiction disorders caused $7 \%$ of all global burden of disease measured in Disability-Adjusted Life Years (DALYs) and $19 \%$ of all years lived with disability in $2016,{ }^{1}$ along with a percentage of $16 \%$ of the world population being affected by mental disorders. ${ }^{1}$ People with mental
Strengths and limitations of this study

- Collaboration with local nurses during interviews to overcome language and cultural barriers.

- Incorporating views from different population groups; patients, family and general practitioners.

- Retrospective study design prone to recall bias.

Selection bias due to purposive sampling.

health disorders are at high risk for disabilities and mortality, ${ }^{2}$ stigma and discrimination. ${ }^{3}$ In most countries, especially low-income and middle-income countries, health systems are inadequately designed to manage mental health problems. ${ }^{24}$ An alarming $76 \%-85 \%$ of people with severe mental disorders in lowincome and middle-income countries do not have access to proper mental healthcare. ${ }^{2}$

One such severe mental disorder, schizophrenia, affects over 21 million people worldwide. The global incidence of schizophrenia is 1.5 per $10000,{ }^{5}$ and the global burden of schizophrenia alone accounts for $1.7 \%$ of Years Lived with Disability (YLDs). ${ }^{4}$ Psychotic symptoms are a core feature of schizophrenia, which is characterised by sensory and/or cognitive disturbances. It has a broad clinical presentation with most prominently symptoms such as hallucinations and delusions, but it can also present itself with symptoms such as thought disorganisation or apathy. ${ }^{6}$ The duration of untreated psychosis, that is, the period between onset of symptoms and initiation of appropriate treatment by a healthcare professional, has been associated with poorer general outcomes. ${ }^{7}$ These manifest themselves as reduced likelihood of remission, poorer social functioning and cognitive impairment. ${ }^{8} 9$ In middle-income and high-income countries several factors playing a role in a prolonged DUP have been identified, such as stigma, ${ }^{10-12}$ the financial 
situation of the patient, ${ }^{11} 13$ illness awareness, ${ }^{11} 1314$ perception, ${ }^{13}$ distance to care facilities ${ }^{15}$ and knowledge of mental illnesses. ${ }^{14}$ Studies in Africa found reasons for low access to mental healthcare related to cultural views on causes of disease and reported people visiting informal healthcare settings first, before reporting to a hospital. ${ }^{16}$ Another recent study found that traditional medicine could contribute to a delay of starting appropriate treatment. ${ }^{13}$ Several countries have conducted research into country-specific factors and have begun to design and test interventions to reduce the DUP. ${ }^{17}$

In Suriname, a middle-income country, limited evidence on psychosis is available. The most recent study on the incidence of schizophrenic disorders was conducted in 2005 and showed an incidence of 1.77 per $10000 .{ }^{18}$ Healthcare is highly centralised, with only one psychiatric hospital located in the capital city Paramaribo. A study researching the equity in healthcare in Suriname reported the use of secondary healthcare to be lower in the rural interior compared with coastal areas. ${ }^{19}$ Suriname is a country in which traditional medicine is widely practised and large parts of the population still rely on these traditional healers in both urban and rural areas. Research in Suriname has shown that within traditional medicine, psychiatric diseases such as depression and anxiety are considered a social imbalance or to have spiritual causes. ${ }^{20}$ These alternate explanations of disease, among other factors, alter help-seeking behaviour of patients and their pathway to care, which seems to be of great importance in psychotic disorders.

Therefore, the aim of this study is to explore the factors related to the duration of untreated psychosis among Surinamese patients, using the perspectives from patients, their families and first-line healthcare professionals in Suriname.

\section{METHODS}

\section{Setting, study design and participants}

In Suriname, the healthcare system is not evenly distributed between rural and urban areas. ${ }^{21}$ It is highly centralised with only one psychiatric hospital based in the capital city Paramaribo, the so-called 'Psychiatric Centre of Suriname' ('PCS'). In addition to this psychiatric hospital, there is one outpatient clinic of the PCS based in Nieuw-Nickerie. With only 10 psychiatrists in place, this calculates to 1 psychiatrist per 56000 population in the country. $^{22}$ To put this into perspective, the Netherlands has a calculated 1 psychiatrist per 4636 population. ${ }^{23}$ General practitioners (GPs) are the first line of care in the formal healthcare system. We conducted semi-structured interviews between February 2019 and April 2019 among patients, family members and GPs. Patients were eligible for inclusion if they (i) signed the informed consent form; (ii) were diagnosed with a psychotic disorder and (iii) were aged between 16 and 65 years of age. Patients were excluded for participation if they (i) had intellectual disability or (ii) had a known drug-induced psychosis.
For family members and GPs no inclusion or exclusion criteria were formulated.

\section{Sampling and materials}

Patients were recruited from various regions in Suriname, namely Paramaribo, Moengo (East), Nieuw-Nickerie (West) and Brownsweg (South). The patients, known by the PCS to have a history of psychosis, were recruited based on the inclusion criteria and contacted by psychiatrists working in the PCS (mainly RN). Eligible patients were selected based on purposive sampling in order to aim at a heterogeneous group of participants concerning area of living and estimated DUP. A convenience sample of family members was used. If patients brought a family member to an interview, this family member was approached for participation in the study. In case patients were visited at home for an interview and a family member was present and willing to participate, this member was interviewed and included in the study. A convenience sample for the GPs was used. GPs were telephonically approached (by $\mathrm{RN}$ ) and asked for participation, and if they were willing to participate they were included. In total, eight GPs were approached for participation in the study. The interviewing process continued until saturation appeared and when no new themes emerged.

A first draft of the topic list was developed by the research team, using topics from previous studies and using the Cultural Formulation Interview, ${ }^{24}$ and the McGill Illness Narrative Interview, ${ }^{25}$ as the foundation of the interview design. Thereafter, it was discussed with PCS healthcare professionals (psychiatrists and nurses) for further improvement and clarification of language, cultural appropriateness and focus on known factors that would be relevant for the research question. This ensured that every factor associated with DUP, insights in the care pathway and cultural factors would be addressed and be understandable to the participants. DUP was defined as the time period between onset of symptoms until initiation of appropriate treatment by a healthcare professional. Onset of symptoms was defined as the first manifestation of symptoms as reported by the patient or their family, irrespective of illness awareness. The treatment initiated by healthcare professionals is based on international evidence-based guidelines. Patients were interviewed about their first episode of psychosis and the topics covered were based on the predicted factors which could influence the DUP. The GP interviews focused on their knowledge of and experience with psychotic disorders. Semi-structured interviews were conducted by (AvB, MdL, LK, MP) in Dutch (the official language of Suriname), with the presence of a nurse from the PCS when possible for any language and communication problems. When patients were only able to speak their local dialect then a nurse would translate. This was the case for 5 $(n=5)$ out of 27 patient interviews and $1(n=1)$ out of 14 family interviews. Doctor interviews were all conducted in Dutch. All interviews were tape recorded and transcribed verbatim. Interview templates for the original and revised 
patient and family interview (online supplemental files 1 ; 2) and GP interview (online supplemental file 3) translated into English can be found in the supplementary materials.

\section{Patient and public involvement}

Patients were first involved in the research process at the stage of conducting the interviews. Experiences from participants, together with themes from previous research, formed the baseline for this study's outcome measures. Patients and public were not involved in the design, recruitment or burden of the study. Research results were shared with the PCS. Participants who showed interest in the study results were recommended to check in with their practising physician on conclusion of the study.

\section{Data analysis}

While conducting and transcribing interviews, data analysis was performed simultaneously. The transcribed interviews were analysed according to thematic analysis. ${ }^{26} \mathrm{In}$ order to ensure reliability, the first four transcripts were independently coded by at least two researchers using a combined inductive and deductive approach. These findings were discussed in order to create a set of codes the four researchers mutually agreed on, and to develop the initial codebook. Subsequently, all other transcripts were coded individually by a researcher. Thereafter, another member of the study team reviewed this coding, adding comments and highlighting possibly overlooked codes. Then discussion followed between the two researchers. The analysis was considered finished when two researchers reached a consensus. The initial codebook was revised based on coding of the subsequent transcripts. When new codes emerged during analysis, these were added to the codebook and previously finished analyses of transcripts were scanned for these newly found codes. Thereafter, the data were refined into themes and subthemes, which was then put into a database. A separate document contained any relevant, supporting quotations.

\section{RESULTS \\ Sociodemographics}

In total, 33 patients were approached for an interview, of which 5 patients were excluded due to having druginduced psychosis $(n=3)$, intellectual disability $(n=1)$ and because of a language barrier which could not be overcome $(n=1)$. Sociodemographic characteristics of one $(\mathrm{n}=1)$ patient were collected through a family interview, because the patient was not able to participate. A total of 13 family members and 8 GPs were included. Sociodemographic characteristics are shown in table 1. Patients had an equal gender distribution and a median age of 39 (IQR 31-48) years. Most patients lived in the capital city Paramaribo. The majority of patients indicated to have multiple ethnicities, and generally, Indian and Creole were the most common. The predominant religions were
Christianity and Hinduism. Most patients were single and lived with their family. The most commonly reached educational level was secondary education. Unemployment was high and most earned under 2000 SRD (€240) per month. The median DUP ( $\mathrm{n}=2$ unknown) was 4 months (IQR 1-36) and the median onset of first symptoms ( $\mathrm{n}=1$ unknown) was 60 months ago (IQR 19-132). Mainly mothers were interviewed, with a median age of 53 (IQR 48-58) years. The majority of GPs were males, with a median age of 47 (IQR 41.5-56) years and they were mainly based in Paramaribo and West-Suriname (table 1). Values were marked 'unknown' in case they could not be reliably retrieved from the interview nor from the medical file.

Using the perspectives of patients, their family members and GPs, we identified various factors related to the duration of untreated psychosis. According to participants, presentation of symptoms and illness awareness, help-seeking behaviour and alternative medicine, social support and stigma, and financial and practical factors are important topics.

\section{Presentation of symptoms and poor illness awareness}

The onset of psychosis was characterised by a wide variety of symptoms. According to patients and family members, hallucinations and disturbed and confused thoughts are the most common first symptoms of a psychosis.

Patient 1: I saw things... on the tv, what I wasn't allowed to see and hear. And I also heard things... and in my thoughts. I thought differently about things... I wanted to have my own thoughts. I had thoughts about things that weren't true.

Nearly half of the patients also presented with aggressive behaviour and agitation. According to a few patients, symptoms of paranoia, sleep disturbance and abnormal motor behaviour were present. Depressive or suicidal thoughts were hardly mentioned (table 2).

Patient 4: It was a sudden outburst (...) that I suddenly became aggressive at home.

Patient 12: The delusions got worse and worse and worse, there were several conspiracies playing in my head and eh I thought people were planning things against me.

According to GPs, patients most frequently present with hallucinations, disturbed and confused thoughts and aggressive behaviour as symptoms of psychosis. They elaborated on disturbed behaviour and indicated seeing a wide range including disobedience, confusion, destruction of property, self-neglect, wandering outside all day, blackmailing and aggression.

GP 6: Aggressive behaviour yes, usually family members come and tell me that a brother or an uncle suddenly behaves aggressively.

The majority of patients expressed that they realised they experienced unusual things, such as hearing voices 
Open access

Table 1 Sociodemographic factors of participants

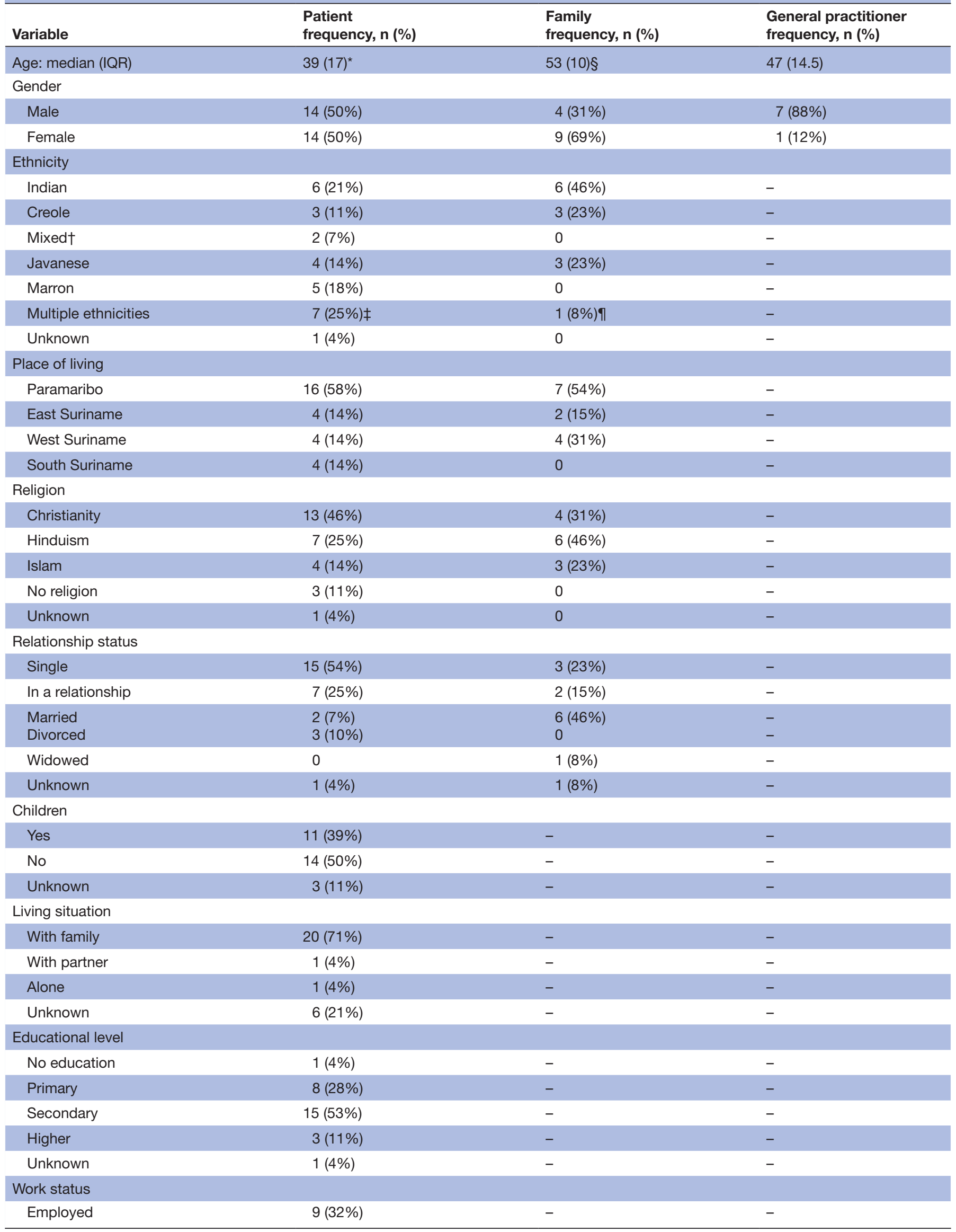


Table 1 Continued

\begin{tabular}{|c|c|c|c|}
\hline Variable & $\begin{array}{l}\text { Patient } \\
\text { frequency, } n(\%)\end{array}$ & $\begin{array}{l}\text { Family } \\
\text { frequency, } \mathrm{n}(\%)\end{array}$ & $\begin{array}{l}\text { General practitioner } \\
\text { frequency, } n(\%)\end{array}$ \\
\hline Unemployed & 19 (68\%) & - & - \\
\hline \multicolumn{4}{|l|}{ Monthly income (SRD) } \\
\hline 0-999 & $19(67 \%)$ & - & - \\
\hline $1000-1999$ & 7 (25\%) & - & - \\
\hline 2000-4999 & $1(4 \%)$ & - & - \\
\hline$>5000$ & 0 & - & - \\
\hline $\begin{array}{l}\text { Unknown } \\
\text { (100 SRD } \approx 12 \text { EUR) }\end{array}$ & $1(4 \%)$ & - & - \\
\hline \multicolumn{4}{|l|}{ Relation to patient } \\
\hline Partner (married) & - & $1(8 \%)$ & - \\
\hline Mother & - & $7(53 \%)$ & - \\
\hline Father & - & $2(15 \%)$ & - \\
\hline Sibling & - & $1(8 \%)$ & - \\
\hline Aunt & - & $1(8 \%)$ & - \\
\hline Adoption mother & - & $1(8 \%)$ & - \\
\hline \multicolumn{4}{|c|}{ Location of GP practice } \\
\hline Paramaribo & - & - & $4(50 \%)$ \\
\hline East Suriname & - & - & 0 \\
\hline West Suriname & - & - & $3(38 \%)$ \\
\hline South Suriname & - & - & $1(12 \%)$ \\
\hline
\end{tabular}

Patient 29 had been older than 62 for years already but did not know their birth date, there was no file. We assigned patient age 62.

*One patient was included in the study based on the story the family told, they did not specify age and therefore this patient was not included in the calculations for age.

†Mixed was not further specified.

$\ddagger$ Multiple ethnicities for patients included the following combinations: Creole-Indigenous-Jewish-Mixed. Creole-Indigenous-Mixed. Creole-

Caucasian-Mixed. Indian-Chinese-Javanese-Caucasian. Indian-Creole (2x). Indian-Jewish-Chinese.

$\S$ Age of three family members unknown.

IIMultiple ethnicities for family included the following combination: Creole-Indigenous-Jewish-Mixed.

GP, general practitioner.

or behaving aggressively. However, they mostly did not seem to associate these experiences with an illness, as they had difficulties realising that their behaviours were abnormal manifestations or that it fell under the scope of a medical problem. Only a few patients associated their symptoms with having an illness.

Table 2 Presenting symptoms reported by participants

\begin{tabular}{lc}
\hline Presenting symptoms & Frequency, n (\%) \\
\hline Disturbed and confused thoughts & $17(61 \%)$ \\
\hline Abnormal motor behaviour & $6(21 \%)$ \\
\hline Hallucinations & $22(79 \%)$ \\
\hline Agitation or aggression & $12(43 \%)$ \\
\hline Delusion or paranoia & $9(32 \%)$ \\
Sleep disturbances & $8(29 \%)$ \\
\hline Depressive or suicidal thoughts & $1(4 \%)$ \\
\hline
\end{tabular}

Presenting symptoms as times reported $(n, \%)$ by patients or their family. Multiple symptoms could apply to one patient. Presenting symptoms mentioned by both patient and family are counted as one.
Patient 18: I never thought of seeking help for voices.

Researcher: Why do you think that it lasted so long until you (...) got help?

Patient 5: Because first I had to think whether it was normal or abnormal.

Most of the patients and family members believed there was a supernatural explanation to the symptoms as they reported the cause of a psychosis to be the cause of a spell or the devil. As there were people from different cultures involved in this study, these supernatural causes varied widely corresponding to patients' their culture or religion. For example, Christians would often mention God or the devil whereas indigenous people would attribute their symptoms to spirits that could do something to you, or could even possess you (Winti). GPs too acknowledged that most patients often hold cultural beliefs as the primary cause for their symptoms.

Patient 14: Some people will say, who tell me that it is the Winti (spirit) from that family. I still talk a language that I do not understand (...) so that is the Winti. 
Patient 1: I thought it's just one of those things they put on you, people in Suriname we believe people put something on you so I thought that was it. I didn't know it was just my illness. Yes, that is Voodoo.

Family 18: We thought it were attacks of the devil.

While some patients attributed their symptoms to other mental or physical factors such as stress or head trauma, a few patients indicated to be completely unaware of their condition at the time.

Patient 10: I was (ill) because I was stressed. I stressed a lot.

Family members reported that they noticed changes in the behaviour of their relatives, and that they associated these changes with an illness. It should be mentioned that participants would also use 'illness' for problems outside of the medical scope. It was rare that neither the family nor other people noticed something was wrong or different about the patient.

Family 7: I could see he was sick, you know? As a mother, you know?

GP 1: Usually the mentors tell us that the patient has been acting strange for a while.

\section{Help-seeking behaviour}

The majority of patients and their family members reported to have visited a traditional healer for alternative care, and this was mainly the first line of care. In Suriname, there is a great variety in who can be classified as a 'traditional healer'. Traditional healers that were repeatedly mentioned by participants of our study were: Pandits, Babas (Hindu culture); Lukuman, Bonuman (Afro-American culture); Chamans (Indigenous culture); Church priests (Christian religion). Some patients indicated they visited a traditional healer multiple times or went to multiple different healers.

Researcher: Why did you first go to the Lukuman (traditional healer)?

Patient 16: Because that... these things spiritual things. (...) This cannot be treated at the general practitioner.

Researcher: How many different people (traditional healers) did you visit in those 4 years?

Patient 6: Almost four.

The second most common first place to seek help was at the GP. Generally, $46 \%$ of the study population saw a GP before receiving treatment at the psychiatric hospital. Most GPs stated that they had been trained in medical school on how to recognise psychotic symptoms and that they would immediately refer patients who present to their clinic with these symptoms. Overall, it was mainly the family that decided on where help should be sought. In a few of these cases, aggressive behaviour was a clear indication for the family that the patient needed immediate medical attention. In the end, in a great majority of cases the family decided to visit a psychiatrist.

Patient 26: They (parents) said ehm, you are confused, go to the doctor.

Researcher: Who decided to go to the GP?

Patient 16: My mother and my grandmother.

Interestingly, some traditional healers also recommended consulting a psychiatrist, according to patients and family members.

Patient 7: That Pandit said you must go to the doctor.

\section{Stigma and social support}

Many participants indicated that there is a negative perception of psychosis and other psychiatric illnesses in society and many patients did not share their problem outside of their families. Often, the Surinamese people would use descriptive words as 'crazy' when referring to someone with a mental illness. Patients expressed fear of being labelled in a negative way.

Researcher: What was for you the main reason not to go to the GP?

Patient 8: That was it, that people will find me crazy.

Researcher: Do people view you differently?

Patient 24: Yes a few of them.

Researcher: Yes? How do they see it?

Patient 24: How should I say it (...), like a madman.

Around half of the study population indicated that stigma was not present within the family and $89 \%$ of participants reported to feel supported by their families. If patients were willing to share their problems, the main first place to do so was with someone within their family. Sharing their problem with outsiders would commonly be done after the patient had already received treatment. Patients noted that family support was often a key component in getting them to seek and receive help. Family members also played a big role in helping patients to stick to their treatment. GPs too emphasised the importance of family support for treatment initiation and continuation.

Family 18: Now when she got these things, I have really, I really did not at all think about getting crazy or being crazy, no (...). But thinking that she is crazy? No, I have not at all thought that.

Patient 6: I have also told my nieces (...) they said; you did the right thing getting help at the Baba (traditional healer). (...) They just want to support you.

Family 7: I go with him, (...), every time he has to come, I come along.

Interviewees also reported a specific stigma on the Psychiatric Centre in Paramaribo. Commonly, it is perceived as a place for mad or crazy people. 
Patient 1: My brother and my mother have said at school I should not tell anything to everybody that I... am under treatment at the PCS. Because then they will treat you different or so.

GP 1: They just don't want to go to the PCS, yes. Because fairly it is seen as a madhouse.

\section{Financial and practical factors around mental health services}

Most patients were insured prior to seeking help, most commonly with basic insurance. Basic insurance covers psychiatric care in Suriname. For those that did not have insurance prior to their symptoms, it was clear that this led to a delay in receiving appropriate treatment. When asked, costs did not seem to keep participants from seeking help. Only very rarely costs were mentioned as an issue for seeking healthcare.

Family 16: We didn't have to pay for it. He has an insurance card.

Researcher: Have you ever doubted whether to seek help because it costs so much money?

Patient 14: No I have never doubted.

Family 7: He had no things, no thing-card.

Nurse: Insurance card.

Family 7: Insurance yes. Then I had to wait a long time (...) three months or so.

None of the interviewees reported any problem with distance to the GP and none mentioned having any difficulty getting help at the GP. The interviewed GPs agreed with this finding and said distance to them was not an issue for patients. Patients living in rural areas did mention that in the past it would be difficult to go to the PCS, situated in Paramaribo, but nowadays it is possible for them to receive psychiatric care through their GPs.

Patient 4: What happened to me, I make time to come to the PCS so for me the distance is no longer important. What is important is I have found help, it is good for me. The distance doesn't matter anymore.

GP 4: The distance I would not say (...) now with these developments we have enough GPs where they can go.

\section{DISCUSSION AND CONCLUSION}

This study aimed to explore the duration of untreated psychosis among Surinamese patients and related factors using the perspectives from patients, their families and first-line healthcare professionals in Suriname. The median DUP was 4 months. Main emerging themes included poor illness awareness, use of alternative medicine, stigma and family support. Patients and families had difficulty recognising symptoms as an illness and would attribute these to supernatural causes, resulting in a delay before contacting a GP or psychiatrist. Significant stigma and fear of being labelled 'crazy' also contributed to a delay in help-seeking, as it withheld people from talking openly about their symptoms. Good family support was associated with a shorter period of time lost before seeing a healthcare professional and often it was the family who took initiative to look for help. Other factors such as financial resources, accessibility to healthcare facilities and knowledge of psychiatric illness among GPs were investigated too, but were found to be of less significant relevance to DUP.

One important and recurrent finding was the lack of illness awareness among the participants and their communities. Many patients and families attributed symptoms to external, predominantly supernatural, causes. These non-medical explanatory models of psychosis have been found previously in non-western countries. ${ }^{27}$ Social, cultural and religious explanations were encountered and would often influence pathway to care. ${ }^{27}$ Likewise, a study in Malawi, ${ }^{28}$ found the sociocultural explanation of witchcraft and spirit possession to be dominant and determinant for subsequent help-seeking. In our study, the most common first place to seek help was traditional medicine, with GP practices following second. Generally, the use of traditional medicines is common in many households in Suriname. ${ }^{29}$ From these results, a relationship between this cultural explanation and alternative medicine as first place to seek help is evident. Various studies in countries with rich cultural history see similar results. ${ }^{28} 3031$ Many patients who reported seeing a traditional healer before seeing a medical specialist, were also the ones who had a longer DUP. A cross-sectional study in South Africa found similar results for contact with traditional healers and DUP. ${ }^{30}$ Burns $e t a \vec{l}^{32}$ too reported an association between alternative medicine and long DUP. There have been promising attempts to set up collaborations between traditional healers and general healthcare,,$^{33}$ or at educating traditional healers for recognising mental diseases. ${ }^{35}$ However, these studies are still juvenile and such a study in Suriname has yet to be performed.

We found that in particular family relations played a major role in social support, help-seeking behaviour and treatment initiation and continuation. Many family members are closely involved in decision-making on the patient's behalf and could therefore be of vital influence on a patients' duration of untreated psychosis. Correspondingly, a US study found patients with stronger family relations to have a shorter DUP compared with individuals who had a more troubled relationship with their families. ${ }^{36}$ In their study, a key component in a strong relationship included open communication, a factor which emerged during our interviews too. Another study from China also found family members misjudging the patients' disease to be the main cause for treatment delay, ${ }^{37}$ again emphasising the importance of family in final decision-making. Family members appear to be the most common initiators in seeking care and to be decision-makers regarding treatment. ${ }^{30} 31$ The social context including family seems to be a promising topic for further research and future interventions aimed at reducing DUP. 
General stigma seemed to be a barrier for patients to talk about their symptoms and seek help, a finding consistent with previous studies. ${ }^{38}$ Recently, Kular et al ${ }^{12}$ found stigma on mental illnesses significantly lengthening DUP. In our study, GPs too attributed treatment delay to stigma. Psychosis is still misunderstood and people are afraid of receiving the label 'crazy'. Correspondingly, we found the Psychiatric Centre in Paramaribo was seen as a place for mad people.

The study is prone to significant recall bias, since all data were gathered through interviews and stories were told in retrospect. The time of onset of first symptoms ranged from 1986 to 2017, from which the recall bias is apparent. During transcript analysis, contradictory information was occasionally found between patients' and their families' stories or between a narrative and the medical file. As there was only one moment of contact, there was no opportunity to clarify contradictory information with the interviewee. In these cases, information from the medical file and subsequently the families' story was deemed more accurate than the patients' story. If conflicting themes occurred within the same transcript, for example, a patient indicating throughout the interview that they were both aware and not aware of their symptoms, both themes were marked as present in the results. This might have led to certain themes being overexposed or underexposed in this research. Additionally, it was difficult to determine DUP for some patients due to inconsistencies between patient and family stories and the medical file. Finally, a selection bias is likely since participants were specifically chosen to create a diverse population.

Strengths of the study were access to patient files to minimise bias for date of admission, onset of symptoms and DUP. Another significant strength is the presence of local nurses during the interviews to overcome language barriers between researcher and interviewee, improving the quality of collected data. The inclusion of GPs strengthens the study by adding a different, medical viewpoint. Furthermore, stories from patients confirmed by family members ensured more reliable data. Lastly, the study design ensured a broad geographical spread for data collection, strengthening the scope to which results can be applied.

In conclusion, multiple factors were related to DUP, of which poor illness awareness, alternative medicine, perceived stigma and social support were predominant. Many other factors were explored, including accessibility to healthcare facilities and financial factors. The importance of factors such as the use of alternative medicine, stigma and family support provide a reflection of the Surinamese society and results of our study facilitate better understanding of this specific population. Knowledge about these country-specific factors can be used as supportive material for measures taken to reduce DUP in Suriname in the future. In concordance with the WHO Traditional Medicine Strategy, ${ }^{39}$ collaboration between traditional healers and the general healthcare system would be a significant step forward and should be pursued.

Acknowledgements The authors would like to thank nurses mw. Saakie, dhr. Moestadjap, dhr. Dameri and dhr. Gadjri for their time, enthusiasm and commitment to this study. The authors would like to thank all the patients and their families for their willingness to meet with them and share their stories. The authors would also like to thank the general practitioners, for freeing up time and providing them with valuable input.

Contributors AvB, MdL, MP and LK initiated this study in collaboration with WV and RN. AvB, MdL, MP, LK, WV and JdZ prepared the research plan and interview templates in the Netherlands. RN and RD were responsible for the ethical approval and provision of appropriate research facilities in Suriname. AvB, MdL, MP and LK performed, transcribed and analysed interviews in Suriname under supervision of $\mathrm{RN}, \mathrm{WV}$ and JdZ. AvB, MdL, MP and LK drafted a first manuscript for the University of Groningen. AvB and JdZ drafted the final manuscript for publication. All authors are responsible for and involved in the project and have critically revised the manuscript. All authors have read and approved the final manuscript. WV is acting as the guarantor of this study.

Funding AvB, MdL, LK and MP each received the UMCG Grant for medical students $(€ 312.50)$ as financial aid for their stay in Suriname.

Competing interests None declared.

Patient consent for publication Not applicable.

Ethics approval Ethical approval was obtained from the Ministry of Public Health in Suriname (VG 20-18). All patients and their families signed an informed consent form prior to the interviews and were informed that participation was voluntary. They were made aware of their right to withdraw from the study at any point and that interviews would be processed anonymously and used for scientific purpose. If participants were unable to write and/or read, they received oral explanation of the study and gave oral consent

Provenance and peer review Not commissioned; externally peer reviewed.

Data availability statement All data relevant to the study are included in the article or uploaded as supplementary information.

Supplemental material This content has been supplied by the author(s). It has not been vetted by BMJ Publishing Group Limited (BMJ) and may not have been peer-reviewed. Any opinions or recommendations discussed are solely those of the author(s) and are not endorsed by BMJ. BMJ disclaims all liability and responsibility arising from any reliance placed on the content. Where the content includes any translated material, BMJ does not warrant the accuracy and reliability of the translations (including but not limited to local regulations, clinical guidelines, terminology, drug names and drug dosages), and is not responsible for any error and/or omissions arising from translation and adaptation or otherwise.

Open access This is an open access article distributed in accordance with the Creative Commons Attribution Non Commercial (CC BY-NC 4.0) license, which permits others to distribute, remix, adapt, build upon this work non-commercially, and license their derivative works on different terms, provided the original work is properly cited, appropriate credit is given, any changes made indicated, and the use is non-commercial. See: http://creativecommons.org/licenses/by-nc/4.0/.

\section{ORCID iD}

Atousa van Beek http://orcid.org/0000-0001-9701-0384

\section{REFERENCES}

1 Rehm J, Shield KD. Global burden of disease and the impact of mental and addictive disorders. Curr Psychiatry Rep 2019;21:10.

2 WHO. Mental health action plan 2013-2020. Geneva, 2013. Available: https://www.who.int/mental_health/publications/action_plan/en/ [Accessed January 2020].

3 United Nations. One in five youth face mental health problems, Secretary General says, calling for attitude change to end stigma in message for international Observance, 2020. Available: https://www. un.org/press/en/2018/sgsm19283.doc.htm

4 Charlson FJ, Ferrari AJ, Santomauro DF, et al. Global epidemiology and burden of schizophrenia: findings from the global burden of disease study 2016. Schizophr Bull 2018;44:1195-203.

5 McGrath J, Saha S, Chant D, et al. Schizophrenia: a concise overview of incidence, prevalence, and mortality. Epidemiol Rev 2008;30:67-76. 
6 Marder S, Davis M. Differential diagnosis and initial management of psychosis in adults - UpToDate, 2017. Available: https://www. uptodate.com/contents/clinical-manifestations-differential-diagnosisand-initial-management-of-psychosis-in-adults?search=psychosis\& source=search_result\&selectedTitle=1 150\&usage_type=default\& display_rank=1\#H261969484

7 Penttilä M, Jääskeläinen $\mathrm{E}$, Hirvonen N, et al. Duration of untreated psychosis as predictor of long-term outcome in schizophrenia: systematic review and meta-analysis. $\mathrm{Br} J$ Psychiatry 2014;205:88-94.

8 Fusar-Poli P, McGorry PD, Kane JM. Improving outcomes of firstepisode psychosis: an overview. World Psychiatry 2017;16:251-65.

9 Reichert A, Jacobs R. Socioeconomic inequalities in duration of untreated psychosis: evidence from administrative data in England. Psychol Med 2018;48:822-33.

10 Sadeghieh Ahari S, Nikpou H, Molavi P, et al. An investigation of duration of untreated psychosis and the affecting factors. J Psychiatr Ment Health Nurs 2014;21:87-92.

11 Hasan AA, Musleh M. Barriers to seeking early psychiatric treatment amongst First-episode psychosis patients: a qualitative study. Issues Ment Health Nurs 2017;38:669-77.

12 Kular A, Perry BI, Brown L, et al. Stigma and access to care in firstepisode psychosis. Early Interv Psychiatry 2019;13:1208-13.

13 Dutta M, Spoorthy MS, Patel S, et al. Factors responsible for delay in treatment seeking in patients with psychosis: a qualitative study. Indian J Psychiatry 2019;61:53-9.

14 Gronholm PC, Thornicroft G, Laurens KR, et al. Mental health-related stigma and pathways to care for people at risk of psychotic disorders or experiencing first-episode psychosis: a systematic review. Psychol Med 2017;47:1867-79.

15 Kvig El, Brinchmann B, Moe C, et al. Geographical accessibility and duration of untreated psychosis: distance as a determinant of treatment delay. BMC Psychiatry 2017;17:176.

16 Burns JK, Tomita A. Traditional and religious healers in the pathway to care for people with mental disorders in Africa: a systematic review and meta-analysis. Soc Psychiatry Psychiatr Epidemiol 2015;50:867-77.

17 Connor C, Birchwood M, Freemantle N, et al. Don't turn your back on the symptoms of psychosis: the results of a proof-of-principle, quasiexperimental intervention to reduce duration of untreated psychosis. BMC Psychiatry 2016;16:127.

18 Selten J-P, Zeyl C, Dwarkasing R, et al. First-contact incidence of schizophrenia in Surinam. Br J Psychiatry 2005;186:74-5.

19 Smits C, Toelsie JR, Eersel M, et al. Equity in health care: an urban and rural, and gender perspective; the Suriname health study. AIMS Public Health 2018;5:1-12.

20 Mans D, Ganga D, Kartopawiro J. Meeting of the minds: traditional herbal medicine in multiethnic Suriname. Aromatic and Medicinal Plants - Back to Nature 2017;2017.

21 WHO. Ministry of health Suriname. WHO-AIMS report on mental health system in Suriname, 2009. Available: https://www.who.int/ mental_health/who_aims_report_suriname.pdf?ua $=1$

22 WHO. Mental health atlas 2017 member state profile Suriname, 2017. Available: https://www.who.int/mental_health/evidence/atlas/ profiles-2017/SR.pdf?ua=1 [Accessed May 2020].
23 Artsenfederatie KNMG. Aantal registraties specialisten/aios KNMG, 2020. Available: https://www.knmg.nl/opleiding-herregistratiecarriere/rgs/registers/aantal-registraties-specialistenaois.htm

24 Dsm-5 Handbook cultural formulation interview, 2015. Available: https://www.dsm-5.nl/documenten/artikel/13/Cultural-FormulationInterview [Accessed Dec 2018].

25 Groleau D, Young A, Kirmayer LJ. The McGill illness narrative interview (mini): an interview schedule to elicit meanings and modes of Reasoning related to illness experience. Transcult Psychiatry 2006;43:671-91.

26 Braun V, Clarke V. Using thematic analysis in psychology. Qual Res Psychol 2006;3:77-101.

27 Bhikha AG, Farooq S, Chaudhry N, et al. A systematic review of explanatory models of illness for psychosis in developing countries. Int Rev Psychiatry 2012;24:450-62.

28 Chilale HK, Silungwe ND, Gondwe S, et al. Clients and carers perception of mental illness and factors that influence helpseeking: where they go first and why. Int J Soc Psychiatry 2017;63:418-25

29 van Andel T, Carvalheiro LG. Why urban citizens in developing countries use traditional medicines: the case of suriname. Evid Based Complement Alternat Med 2013;2013:1-13.

30 Tomita A, Burns JK, King H, et al. Duration of untreated psychosis and the pathway to care in KwaZulu-Natal, South Africa. J Nerv Ment Dis 2015;203:222-5.

31 Omer AA, Mufaddel AA. Attitudes of patients with psychiatric illness toward traditional healing. Int J Soc Psychiatry 2018;64:107-11.

32 Burns JK, Jhazbhay K, Kidd M, et al. Causal attributions, pathway to care and clinical features of first-episode psychosis: a South African perspective. Int J Soc Psychiatry 2011;57:538-45.

33 Veling W, Burns JK, Makhathini EM, et al. Identification of patients with recent-onset psychosis in KwaZulu natal, South Africa: a pilot study with traditional health practitioners and diagnostic instruments. Soc Psychiatry Psychiatr Epidemiol 2019;54:303-12.

34 Gureje O, Appiah-Poku J, Bello T, et al. Effect of collaborative care between traditional and faith healers and primary health-care workers on psychosis outcomes in Nigeria and Ghana (COSIMPO): a cluster randomised controlled trial. Lancet 2020;396:612-22.

35 Mbwayo AW, Ndetei DM, Mutiso V, et al. Traditional healers and provision of mental health services in cosmopolitan informal settlements in Nairobi, Kenya. Afr J Psychiatry 2013;16:134-40.

36 Hernandez M, Hernandez MY, Lopez D, et al. Family processes and duration of untreated psychosis among US Latinos. Early Interv Psychiatry 2019;13:1389-95.

37 Qiu Y, Li L, Gan Z, et al. Factors related to duration of untreated psychosis of first episode schizophrenia spectrum disorder. Early Interv Psychiatry 2019;13:555-61.

38 Hasan A. Determinant of treatment delay in the first episode of psychosis: a qualitative study. J Psychol Clin Psychiatry 2018;9:258-63.

39 WHO. Who traditional medicine strategy: 2014-2023, 2013. Available: https://www.who.int/medicines/publications/traditional/ trm_strategy14_23/en/ [Accessed Sep 2020]. 